\title{
2021 European Thyroid Association Guidelines for the Management of lodine-Based Contrast Media-Induced Thyroid Dysfunction
}

\author{
Tomasz Bednarczuk ${ }^{a}$ Thomas H. Brix ${ }^{b}$ Wolfgang Schima ${ }^{c}$ Georg Zettinig ${ }^{d}$ \\ George J. Kahaly \\ ${ }^{a}$ Department of Internal Medicine and Endocrinology, Medical University of Warsaw, Warsaw, Poland; ${ }^{b}$ Department \\ of Endocrinology and Metabolism, Odense University Hospital, Odense, Denmark; 'Department of Diagnostic and \\ Interventional Radiology, Goettlicher Heiland Krankenhaus, Barmherzige Schwestern Krankenhaus, and Sankt \\ Josef Krankenhaus, Vienna, Austria; ${ }^{d}$ Schilddruesenpraxis Josefstadt, Vienna, Austria; eDepartment of Medicine I, \\ Johannes Gutenberg University (JGU) Medical Center, Mainz, Germany
}

\section{Keywords}

lodine $\cdot$ Contrast media $\cdot$ Hyperthyroidism $\cdot$ Hypothyroidism . Multinodular goiter

\begin{abstract}
Given the fact that a large number of radiological examinations using iodine-based contrast media (ICM) are performed in everyday practice, clinicians should be aware of potential ICM-induced thyroid dysfunction (TD). ICM can induce hyperthyroidism (Hyper) or hypothyroidism (Hypo) due to supraphysiological concentrations of iodine in the contrast solution. The prevalence of ICM-induced TD varies from 1 to $15 \%$. ICM-induced Hyper is predominantly found in regions with iodine deficiency and in patients with underlying nodular goiter or latent Graves' disease. Patients at risk for ICMinduced Hypo include those with autoimmune thyroiditis, living in areas with sufficient iodine supply. Most cases of ICM-induced TD are mild and transient. In the absence of prospective clinical trials on the management of ICM-induced TD, an individualized approach to prevention and treatment, based on patient's age, clinical symptoms, preexisting thyroid diseases, coexisting morbidities and iodine intake must be advised. Treatment of ICM-induced Hyper
\end{abstract}

with antithyroid drugs (in selected cases in combination with sodium perchlorate) should be considered in patients with severe or prolonged hyperthyroid symptoms or in older patients with underlying heart disease. It is debated whether preventive therapy with methimazole and/or perchlorate prior to ICM administration is justified. In ICM-induced overt Hypo, temporary levothyroxine may be considered in younger patients with symptoms of Hypo, with an underlying autoimmune thyroiditis and in women planning pregnancy. Additional clinical trials with clinically relevant endpoints are warranted to further aid in clinical decision-making in patients with ICM-induced TD. O 2021 European Thyroid Association Published by S. Karger AG, Basel

\section{Introduction}

During the past decades, the number of radiological examinations, particularly computed tomography (CT) and vascular interventional procedures, which require iodine-based contrast media (ICM) has risen dramatically [1]. Thus clinicians must be aware of possible adverse effects (Contrast Media Safety Guidelines 10.0; http://
Correspondence to:

Tomasz Bednarczuk, tbednarczuk@wum.edu.pl 
Table 1. Current nonionic iodine-based contrast agents for parenteral administration [8]

\begin{tabular}{llllll}
\hline $\begin{array}{l}\text { Chemical } \\
\text { name }\end{array}$ & $\begin{array}{l}\text { Monomer } \\
\text { or dimer }\end{array}$ & $\begin{array}{l}\text { Iodine content, } \\
\text { range, } \mathrm{mg} / \mathrm{mL}\end{array}$ & $\begin{array}{l}\text { Osmolality, } \\
\mathrm{mOsm} / \mathrm{kg} \mathrm{H} \mathrm{O}\end{array}$ & $\begin{array}{l}\text { Osmolality } \\
\text { type }\end{array}$ & $\begin{array}{l}\text { Viscosity, mPa.s } \\
\text { at } 37^{\circ} \mathrm{C}\end{array}$ \\
\hline $\begin{array}{l}\text { Iohexol } \\
\text { Iopamidol }\end{array}$ & $\begin{array}{l}\text { Monomeric } \\
\text { Monomeric }\end{array}$ & $180-350$ & 640 & Low osmolality & 6.1 \\
Iopromide & Monomeric & $150-370$ & 616 & Low osmolality & 4.5 \\
Ioversol & Monomeric & $240-350$ & 590 & Low osmolality & 4.7 \\
Iobitridol & Monomeric & $250-350$ & 645 & Low osmolality & 5.5 \\
Iomeprol & Monomeric & $150-400$ & $521 \pm 24$ & Low osmolality & 6.0 \\
Iodixanol & Dimeric & $270-320$ & 290 & Low osmolality & $4.5 \pm 0.4$ \\
\hline
\end{tabular}

Osmolality is given for an iodine content of $300 \mathrm{mg} / \mathrm{mL}$ (monomeric agents) and for $320 \mathrm{mg} / \mathrm{mL}$ (iodixanol), respectively.

www.esur.org/esur-guidelines/). Although certain adverse effects (e.g., contrast-induced nephropathy) have been widely studied, the effects of ICM on the thyroid gland remain poorly characterized. It is generally accepted that ICM can cause thyroid dysfunction (TD) because of supraphysiological concentrations of iodine in the contrast solution [2-5]. While the normal thyroid gland can usually adapt to an excess iodine load, individuals with an underlying thyroid disorder may develop hyperthyroidism (Hyper) or hypothyroidism (Hypo).

When it comes to radiological examinations using ICM in patients with thyroid disorders specialists from different fields of medicine are regularly faced with a dilemma. This document reviews current evidence and provides answers to questions commonly asked in clinical practice concerning the potential risk, prevention, diagnosis, and management of ICM-induced TD.

\section{Overview of Radiographic ICM}

Current radiographic (X-ray and CT) imaging uses electromagnetic radiation that penetrates the body. Contrast agents may be used with these techniques to enhance the differences seen between body tissues [6]. Among the ICM, 2 different types have to be distinguished: ionic and nonionic compounds. Ionic contrast agents with high osmolality are not in use for parenteral administration anymore, but only for oral or rectal application, which does not result in significant systemic uptake. Only nonionic iodine-based contrast agents are used for parenteral (intravenous [IV] or intra-arterial) administration in radiologic examinations. These agents are either monomeric or dimeric tri-iodinated benzene rings with an organic functional group [7]. The preference for iodine over metal ions with high atomic numbers is based on its high ef- ficacy in absorption of X-rays, the stable binding of multiple iodine atoms to an organic molecule, and the low toxicity of ICM [8].

\section{Physicochemical Properties}

Non-ionic ICM is hydrophilic, which is a prerequisite for intravascular injection of larger quantities. Low viscosity of contrast agents is necessary for rapid administration during CT or angiography [8]. Viscosity depends on chemical structure, iodine content, and temperature of contrast agent: dimeric agents are more viscous than monomeric agents (Table 1). In general, higher iodine content and lower temperature result in higher viscosity [9].

\section{Contrast Material Dosage}

Iodine-based contrast agents are mostly administered parenterally during CT, angiography, or IV urography, and to a lesser extent orally, enterally (during endoscopic retrograde cholangiopancreatography), rectally, or intracavitary. The amount of contrast material delivered during CT ranges normally between 60 and $150 \mathrm{~mL}$, depending on the indication, the scanned region, and the scan range, as well as the body weight [10-12]. Given the standard iodine concentration of $300 \mathrm{mg} / \mathrm{mL}$ then a total amount of $18-45 \mathrm{~g}$ of iodine is administered during contrast-enhanced CT. With catheter angiography, even higher doses of contrast may be used [7].

\section{Prevalence of ICM-Induced TD}

At present, the prevalence and clinical significance of ICM-induced TD is incompletely characterized. Recent large retrospective observational studies confirmed an association between ICM exposure and the develop- 
ment of TD. In a nested case-control study using data from the Partners Health-Care Research Patient Data Registry (USA), ICM exposure was associated with incident overt Hyper (oHyper) (defined as a suppressed TSH $<0.1 \mathrm{mIU} / \mathrm{L}$; odds ratio [OR], 2.50; 95\% CI, $1.06-$ 5.93) and with incident overt Hypo (oHypo) (defined as elevated serum TSH >10 mIU/L; OR, 3.05; 95\% CI, 1.078.72) [13]. A significantly higher risk of ICM-induced TD (based on International Classification of Diseases, Ninth Revision, Clinical Modification codes) was demonstrated in Taiwan among 1 million patients in the general population over a single year time period; the adjusted hazard ratio (HR) of Hyper and Hypo compared with controls was 1.22 (95\% CI, 1.04-1.44) and 2.00 (95\% CI, 1.65-2.44), respectively [14]. Moreover repeated ICM exposure ( $>1$ time of ICM per year) increased significantly the risk of thyroid disorders ( $\mathrm{HR}=$ 3.04; 95\% CI, 2.47-3.73) [15]. A subsequent study using the Taiwan's National Health Insurance Research Database found that patients with euthyroid nodular goiter had almost 5-fold higher risk of ICM-induced TD than patients without thyroid nodule(s), including Hyper (HR 5.77, CI 2.64-12.62) and Hypo (HR 4.95, CI 2.1511.40) [16]. A retrospective cohort study of children aged younger than 4 years in the Kaiser Permanente Northern California (USA) database revealed that the incidence density ratio for Hypo in ICM-exposed patients was 1.33 per 1000 person months (95\% CI, 0.91.8). It was highest among children younger than 3 months old [17]. A further single-institution case-control study in the USA confirmed that ICM exposure increases the risk of incident Hypo in pediatric patients (OR, 2.60; 95\% CI, 1.43-4.72) [18].

Despite convincing retrospective studies, the results of prospective observational studies in various countries examining changes in thyroid hormone levels following a single exposure to ICM are highly variable (online suppl. Table 1; see www.karger.com/doi/10.1159/000517175 for all online suppl. material) $[19,88-91]$. The prevalence of ICM-induced Hyper ranged from 0 to $9 \%$, being greater in countries with iodine deficiency. ICM-induced Hypo was more prevalent in countries with a high iodine intake and occurred in up to $15 \%$ of patients. These findings must be interpreted with caution due to important limitations, for example, (i) substantial heterogeneity between studies was present with respect to both groups of patients (unselected patients vs. patients without previous TD) as well as radiological examination (coronary angiography, CT, hysterosalpingography) using different ICM; (ii) most studies were of small size applying differ- ent follow-up periods; (iii) heterogeneity with respect to definition of TD; and (iv) an in-depth analysis of relevant clinical measures is generally missing.

\section{Methodology}

Given the controversies regarding the prevalence, clinical significance, prevention, and treatment of ICM-induced TD, in 2019 T.B., T.H.B., W.S., G.Z., and G.J.K. asked the European Thyroid Association (ETA) to form a task force to provide practice guidelines for the management of ICM-associated TD. Membership on the panel was based on clinical expertise, scholarly approach, representation of endocrinology, nuclear medicine, and radiology, as well as ETA membership. The proposed task force was approved by the ETA Guidelines Board and the ETA Executive Committee.

The present international guidelines concerning various TD do not address the problem of ICM exposure in detail [20-23]. Moreover, a recent survey performed in Norway suggested a need of optimization of routines regarding ICM administration to patients at risk for TD [24]. The aim of the task force was to offer an evidence-based, up-to-date, and thorough document critically encompassing and evaluating the current available scientific information worldwide.

\section{Literature Search}

Relevant published articles were identified by searching PUBMED (www.ncbi.nlm.nih.gov). Furthermore, all references were evaluated for any additional appropriate citation.

Grading

The task force rated the recommendations according to the GRADE system, which was used in the previous guidelines issued by the ETA [25-27]. The following coding system was used: (1) indicates a strong recommendation (for or against) because the benefits outweigh the risks and is associated with the phrase "we recommend"; (2) denotes a weak recommendation (for or against) in which the treatment depends on the patient's preference because the benefits and risks were uncertain and are associated with the phrase "we suggest." Evidence grading: ØOOO denotes very low-quality evidence; $\varnothing \varnothing \mathrm{O}$, low; $\varnothing \varnothing \varnothing \mathrm{O}$, moderate; and $\varnothing \varnothing \varnothing \varnothing$, high quality.

\section{Recommendations}

Recommendations before Radiological Examinations Using ICM

Screening of Baseline Thyroid Function prior to ICM Exposure in the General Population

In the general population, clinically evident TD is a rare complication after ICM exposure. The number needed to harm for ICM-induced Hyper and Hypo was one in every 250 patients [14]. These results were confirmed by single-institution retrospective analyses and a recent metanalysis [19]. In the Netherlands, 7 patients with nod- 
ular goiter needed hospitalization between 1987 and 1990 due to clinically severe ICM-induced Hyper among a cohort of 24,000 CT scans [28]. More recently in Denmark, 14 patients (6\% of all new cases of Hyper) had an ICM injection within 2 years before the hyperthyroid episode. During the same period, 30,000-35,000 injections of ICM were given for CT only [29].

\section{Recommendations}

1. We do not recommend baseline thyroid function testing of the general population before radiological examinations using ICM administration. 1, ØØОО

Screening of Baseline Thyroid Function in High-Risk Patients for ICM-Induced TD

It is clinically relevant to detect undiagnosed TD prior to ICM exposure. In areas of iodine deficiency, the prevalence of undiagnosed Hyper may be surprisingly high. In a large prospective study in Italy, $7.2 \%$ of patients without known thyroid disease had Hyper (predominantly subclinical Hyper [SHyper]) prior to elective coronary angiography [30]. In a second large Italian study including 1752 consecutive patients before coronary angiography [31], TD was a frequent condition involving about $40 \%$ of the population as follows: low T3 syndrome - 28\%, Hypo (defined as increased serum TSH)- 10\% and Hyper (decreased TSH) - $2 \%$. Further, a retrospective study revealed that a substantial proportion of inpatients (45\%) with undiagnosed thyrotoxicosis received IV ICM prior to endocrine evaluation [32]. These results suggest that an aggressive case-finding approach, based on identifying those persons most likely to have TD prior to ICM examinations might be beneficial [33].

Several risk factors have been associated with ICMinduced TD (Table 2) [3, 5, 82-87]. Although ICM-induced TD can occur in any individual, it is most commonly reported in patients with an underlying thyroid disease. The most frequently reported risk factor is nodular goiter [16]. High-risk patients for ICM-induced Hyper include those with nodular goiter containing autonomously functioning thyroid tissue and/or latent Graves' disease. ICM-induced Hypo is predominantly found in regions with sufficient iodine supply and in patients with autoimmune thyroiditis.

In patients at high-risk for Hyper, diagnostically equivalent alternative imaging modalities, not requiring ICM should be considered, foremost ultrasound, or much less frequently scintigraphy, MRI, or unenhanced CT. Some centers suggest that the knowledge of an existing TD (at least serum TSH) prior to a contrast-enhanced study may be helpful, especially in the elderly with underlying cardiovascular diseases. However, the benefits of TSH screening in high-risk patients have not been demonstrated so far. Moreover, a normal baseline thyroid function does not exclude the development of TD after ICM exposure [34]. It may be explained by the fact that many patients with autonomously functioning thyroid nodules may have a normal serum TSH value [35].

\section{Recommendations}

2. Prior to ICM exposure, we suggest a thorough casefinding approach, based on identifying those persons most likely to have undiagnosed TD. 2, ØØОО

3. We suggest measurement of baseline serum TSH in high-risk patients for ICM-induced TD, especially in the elderly and subjects at risk for cardiovascular diseases. If serum TSH is abnormal, thyroid hormones (T3 and/or T4) should be measured. 2, ØОOO

\section{Is Baseline TD a Contraindication to ICM}

Administration?

OHyper is generally considered to be a contraindication to radiological examinations using ICM (as stated in the drug insert) and whenever possible the search for alternative imaging modalities is recommended. In case of emergency/no alternative radiological modality is possible; treatment with antithyroid drug (ATD) should be initiated prior to radiological study using ICM (combination therapy with ATD and perchlorate may be considered in selected cases). The European Society of Urogenital Radiology questionnaire for ICM administration, which should be completed by the referring clinician, includes a question about Hyper (http://www.esur. org/esur-guidelines/). However, the effect of ICM administration in patients with untreated OHyper and in patients treated with ATD is unpredictable, and in severe cases, it may lead to life-threatening complications (online suppl. Table 2) [36-38]. On the other hand, ICM administration does not always lead to exacerbation of OHyper [30].

Patients with persistent endogenous SHyper should be diagnosed and managed according to the international/ local guidelines [25]. Establishing the etiology of persistent SHyper is important in risk stratification of ICMinduced Hyper. Patients with pre-existing functional thyroid autonomy by scintigraphy using Tc-99m-pertechnetate/iodine-123 are at risk of developing OHyper, whereas patients with SHyper without thyroid autonomy have a negligible risk of ICM-induced Hyper [39]. Prior to both, elective and emergency radiological studies, an 
Table 2. Risk factors for iodine-induced thyroid dysfunction

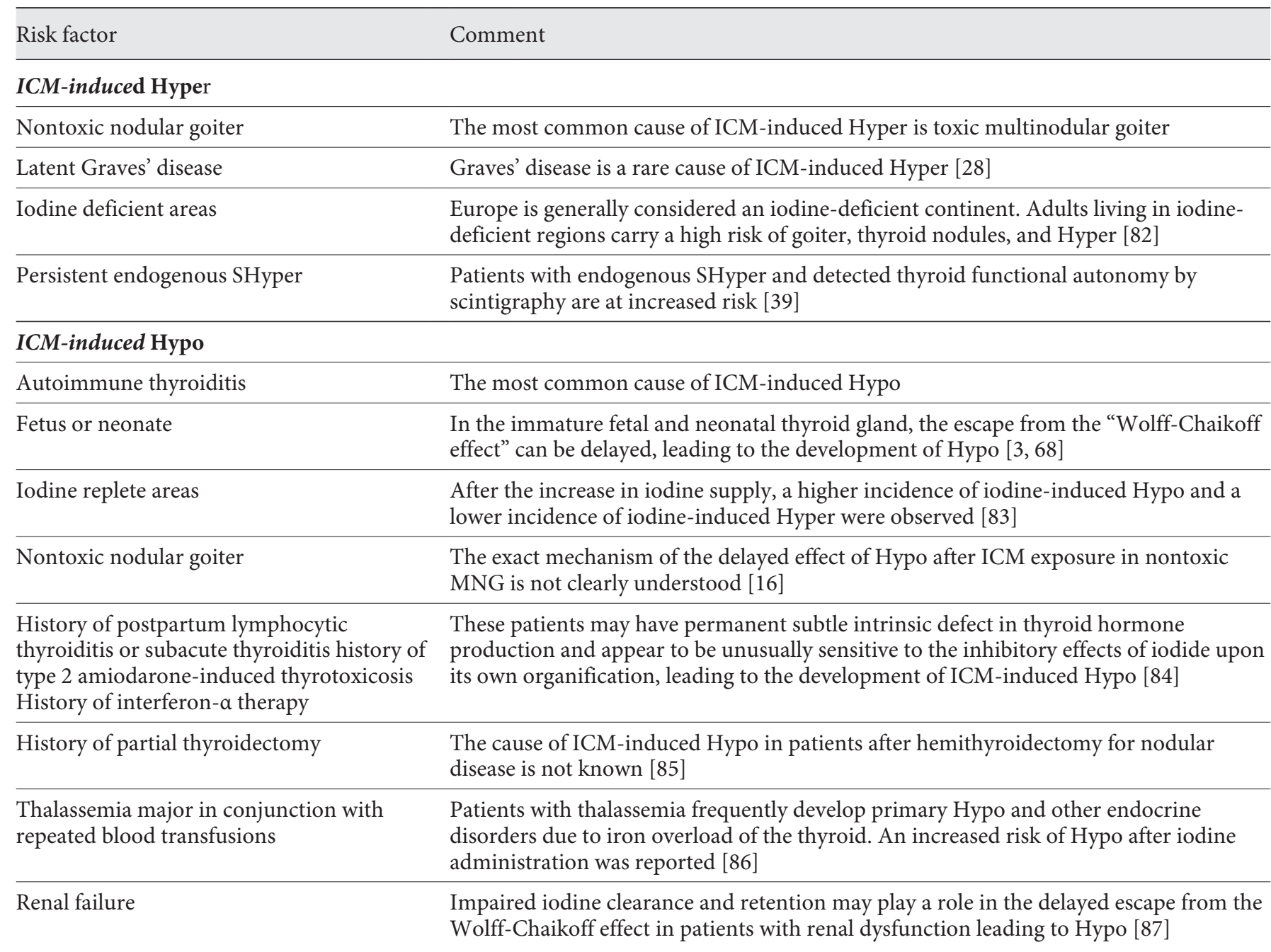

ICM, iodine-based contrast media; Hyper, hyperthyroidism; Hypo, hypothyroidism.

endocrinologic consultation and initiation of preventive medication may be considered.

Baseline subclinical Hypo (SHypo) and OHypo are not a contraindication to ICM administration. Patients with OHypo and SHypo should be diagnosed and managed according to the local guidelines $[21,22]$. Due to possible exacerbation of Hypo, close monitoring may be advised in selected patients after ICM administration.

\section{Recommendations}

4. We do not recommend radiological examinations using ICM in patients with OHyper. Alternative imaging procedures (e.g., ultrasound, MRI, noncontrast CT) should be considered. $1, \varnothing \varnothing$
5. Persistent endogenous SHyper is not a contraindication to radiological examinations using ICM. Prior to elective radiological studies, we recommend to establish the etiology of SHyper. Endocrine consultation may be advised. $1, \varnothing \varnothing \varnothing \mathrm{O}$

6. Baseline untreated SHypo and OHypo are not a contraindication to ICM administration. Due to possible exacerbation of Hypo, close monitoring after ICM is suggested in selected patients. 2, ØØОО

7. Patients on thyroid hormone replacement are not at risk for ICM-induced TD and do not require specific management. $1, \varnothing 000$ 
Fig. 1. Prophylactic treatment in high-risk patients for ICM-induced Hyper. ICM, iodine-based contrast media; Hyper, hyperthyroidism.

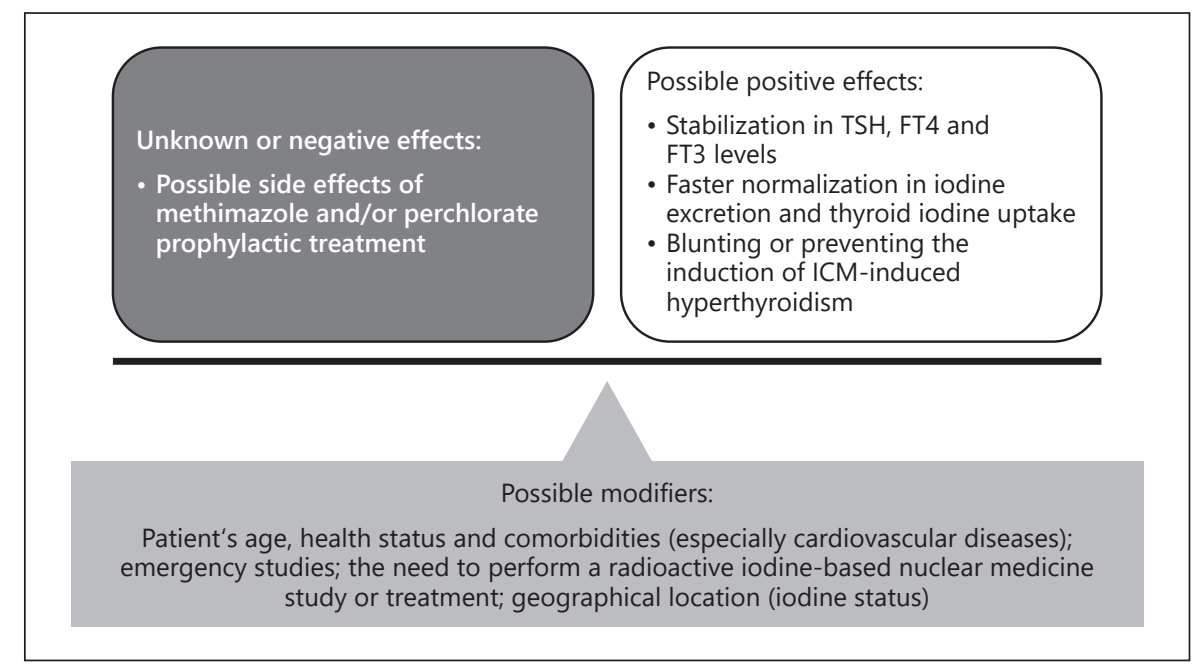

Should High-Risk Patients for ICM-Induced Hyper

Receive Prophylactic Treatment before a Radiological Examination?

The management of patients with a high risk for ICMinduced Hyper (especially patients with persistent endogenous SHyper) remains controversial [20]. After ICM administration, clinical symptoms of Hyper and/or thyroid function tests should be monitored. It is debated whether short-term pretreatment with ATDs (inhibition of thyroid hormone synthesis) and/or perchlorate (inhibition of iodine uptake) is justified to decrease the risk of incident thyrotoxicosis.

Only a few prospective studies (including only one randomized clinical trial) on efficacy of prophylactic treatment exist, most of these were performed in Germany and Austria. Short-term therapy with $60 \mathrm{mg}$ of methimazole and $1 \mathrm{~g}$ of sodium perchlorate $(24 \mathrm{~h}$ before and on the day of coronary angiography) led to a reduced iodine load of the thyroid when monitored by scintigraphy and urine iodine excretion 4 and 12 weeks after angiography. One of 27 (3.7\%) treated patients versus 3 of 33 (9\%) control patients developed Hyper [40]. These results are in line with other findings [41] that intrathyroidal iodine concentration (by means of X-ray-fluorescenceanalysis) did not change in the group of volunteers on perchlorate medication after ICM administration. The protective effect of a monotherapy with either $20 \mathrm{mg}$ of methimazole per day or $900 \mathrm{mg}$ of perchlorate per day (starting 1 day before coronary angiography and lasting for 14 days) was studied in 51 patients with endogenous SHyper with functional thyroid autonomy [42]. Although mild OHyper occurred in both treatment and control groups ( 2 in the control group vs. one in each of the treated groups), the authors observed stable serum TSH, FT4, FT3 levels, normal iodine urine excretion, and normal thyroid uptake 30 days after ICM administration in the treated groups. These results were confirmed in 19 patients undergoing coronary angiography, with endogenous SHyper and functional thyroid autonomy, who were treated with perchlorate alone $(900 \mathrm{mg} /$ day, divided into 3 doses, for 2 weeks) or combined with methimazole (20-60 mg for 1-2 weeks). Mild Hyper was observed in 2 out of 19 patients, whereas in the remaining 17 patients, thyroid hormones (FT4 and FT3) levels decreased and TSH levels increased 28 days after coronary angiography [39].

The results of studies analyzing the prophylactic treatment prior to ICM administration in high-risk patients are shown in Figure 1. The treatment may be effective in reducing iodine load of the thyroid, stabilizing the thyroid function, and blunting/preventing ICM-induced OHyper. On the other hand, both drugs (methimazole and perchlorate) have a significant rate of side effects. Unfortunately, the prophylactic treatment does not prevent ICM-induced Hyper in all cases. Most importantly, an in-depth analysis of relevant short- and long-term clinical measures of therapeutical success besides surrogate markers (serum thyroid hormone levels, iodine urine excretion, and iodine thyroid uptake) is missing. Therefore, the final decision should be individualized with respect to pre-existing thyroid diseases, coexisting morbidities (especially cardiovascular diseases), patient's age, iodine intake, and the need to perform radioiodine (RAI)-based studies. 
The published treatment protocols vary substantially. The Austrian Thyroid Association recommends using perchlorate as preventive treatment $(600 \mathrm{mg}$ prior to examination and continuation with $3 \times 300 \mathrm{mg}$ daily for 7-14 days) in high-risk patients before emergency studies [43]. Prior to ICM exposure, a consultation in a Thyroid Clinic is advised. In many countries, the following ATD regimen is used: methimazole $20-30 \mathrm{mg}$ daily, starting the day before ICM administration and continuing for 14 days [2]. A few authors suggested that a combination therapy of methimazole and perchlorate may be more effective $[34,42]$.

\section{Recommendations}

8. We do not recommend prophylactic treatment in all patients at high-risk for developing ICM-induced Hyper. However, patients at risk for Hyper should be monitored after ICM injection. 1, ØØОО

9. We suggest considering prophylactic treatment with methimazole and/or perchlorate before emergency radiological examinations in selected patients who are less tolerant to the effects of ICM-induced Hyper, for example, elderly with persistent endogenous SHyper and/or nodular goiter, and/or concomitant cardiovascular disease, especially in areas of iodine deficiency. 2, ØØ०О

\section{Monitoring of Patients after Radiological \\ Examinations Using ICM}

Does ICM Exposure Affect Thyroid Function Tests

in Euthyroid Subjects?

The most rapid (hours to days) effect of pharmacologic doses of iodine is to inhibit organification of iodide, thereby diminishing hormone biosynthesis (Wolff-Chaikoff effect) and decreasing thyroglobulin proteolysis, thereby decreasing thyroid hormone secretion $[3,5]$. The resulting small decrease in serum $\mathrm{T} 4$ and $\mathrm{T} 3$ levels causes a compensatory, transient increase in TSH concentrations $[44,45]$. After the acute phase, normal thyroid hormone synthesis resumes, even with continued excess iodide exposure, a phenomenon described as the escape from the acute Wolff-Chaikoff effect. However, weeks after ICM exposure, several prospective observational studies revealed an increase or decrease in serum TSH, FT4, and FT3 levels, dependent probably on the iodine intake and pre-existing thyroid disease [46]. While these changes are within the reference and in most untreated cases, they may interfere in the proper interpretation of thyroid function tests [47].

\section{Recommendations}

10. ICM administration may cause transient changes in serum TSH, T4, and T3 levels in healthy individuals, usually within the reference values. While interpreting thyroid function tests, we suggest taking ICM exposure (within the last 1-3 months) into consideration. 2, ØØØ०

\section{Should Thyroid Function Be Monitored after ICM}

\section{Administration?}

There is no indication for routine monitoring for ICM-induced TD because this would not be cost-effective $[13,14]$. Therefore, all patients should be informed that signs and symptoms of Hyper or Hypo may occur after ICM administration and should be advised to alert the medical team if such develop.

Routine thyroid function testing is favored by a few experts in older patients with a high risk of ICM-induced Hyper and patients with an underlying unstable cardiovascular disease. In such cases, measurement of serum TSH (and if low, T4 and T3) 3-4 weeks after ICM exposure is advised. Routine screening for ICM-induced Hypo may be also considered in selected high-risk patients, especially in women planning pregnancy after hysterosalpingography with oil-soluble ICM and in neonates [4850].

\section{Recommendations}

11. We do not recommend routine TSH monitoring following ICM administration. 1, ØØØO

12. The exact timing of the ICM-TD is often unpredictable. We suggest advising patients to alert the medical team if they develop signs and symptoms of TD. 2, $\varnothing 000$

13. We suggest measuring thyroid function 3-4 weeks after ICM exposure in high-risk patients for ICM-induced Hyper, especially in the elderly and/or those with an underlying unstable cardiovascular disease. 2 , $\varnothing \varnothing 00$

When can Isotope Imaging of the Thyroid or RAI Treatment Be Performed after ICM Exposure?

The administration of ICM is known to suppress thyroidal RAI uptake, lasting for several weeks [51]. Some studies on urinary iodine secretion after ICM administration for outpatient CT scans indicate that $75 \%$ of patients' values returned to baseline within 5-6 weeks and 90\% within 11 weeks $[46,52]$. A study performed in post-thyroidectomy patients requiring RAI treatment demonstrated that 1 month is sufficient for urinary iodine to 
return to its baseline value after the use of ICM [53]. These results may be used to guide the timing of RAI treatment as well as diagnostic scintigraphy with radioactive iodine or Tc-99m-pertechnetate following contrast exposure. The American Thyroid Association Management Guidelines for Adult Patients with Thyroid Nodules and Differentiated Thyroid Cancer (2015) state that concerns about iodine burden from IV contrast agents causing a clinically significant delay in subsequent wholebody scans or RAI treatment post-thyroidectomy is generally unfounded [54]. In doubtful situations, a spot or 24-h urinary iodine level may be checked before isotope studies. It should be remembered that oil-soluble ICM circulates for longer than other water-soluble media [48, $55,56]$.

\section{Recommendations}

14. We recommend avoiding isotope imaging of the thyroid and radioactive iodine treatment for one to 2 months after ICM injection. 1, ØØØO

\section{Diagnosis and Management of ICM-Induced Hyper \\ Establishing the Diagnosis and Etiology of ICM-Induced Hyper}

Generally, the diagnosis of ICM-induced Hyper is based on: (i) the clinical manifestations of Hyper, (ii) laboratory findings, and (iii) history of ICM exposure. Although ICM-induced Hyper may occur up to 1 year after a radiological examination with ICM $[13,16]$, the majority of patients develop symptoms and/or signs of Hyper within 3-10 weeks after exposure [30]. The severity of ICM-induced Hyper is unpredictable. The majority of patients remain oligo- or asymptomatic. However, the consequences of even mild ICM-induced Hyper should not be underestimated in geriatric patients and in patients with unstable cardiovascular status; it can aggravate pre-existing cardiac diseases leading to atrial fibrillation, congestive heart failure, worsening of angina pectoris, and thromboembolism [57-59, 92-98]. Finally in rare cases, ICM exposure may lead to thyroid storm [36-38] (online suppl. Table 2).

The diagnosis of Hyper is confirmed by thyroid function tests (TSH, FT4 and TT3 or FT3). In most patients, the history of ICM exposure is apparent. In doubtful situations, checking urinary iodine concentration (spot or 24-h sampling) may be helpful to support iodine excess as the cause of Hyper and to monitor treatment.

ICM-induced Hyper is not a single etiological entity $[3,5]$. It is generally accepted that ICM-induced Hyper can develop due to pre-existing areas of functional au- tonomy within the thyroid. Upon exposure to excess iodine, the autonomous areas produce thyroid hormone in excess and independent of autoregulatory mechanisms (the Jod-Basedow phenomenon). The most important risk factors include nodular thyroid disease (most common cause), latent Graves' disease, and long-standing iodine deficiency. Very rarely, ICM-induced Hyper has been reported in patients without underlying thyroid disease (ICM-induced thyroiditis) [60]. Moreover, other causes of Hyper must also be considered, since Hyper may not be related to the iodine exposure. Therefore, an evaluation to determine the etiology of Hyper is important based on international/local guidelines [25, 27]. Diagnostic serological and imaging testing can include the following, depending on available expertise and resources: (i) measurement of TSH-receptor antibodies [61, 62], (ii) thyroid ultrasonography assessing nodularity, echogenicity of the parenchyma, and blood flow, and (iii) thyroid scintigraphy. The results of a RAI or $99 \mathrm{~m}$-pertechnetate thyroid scan (which is in other clinical settings the gold standard for differential diagnosis between hyperfunctioning thyroid tissue and thyroid cell destruction) should be interpreted here with caution because a low isotope uptake may be seen up to 2 months after ICM exposure. However, a single ICM exposure rarely reduces the uptake to $<1 \%$ as it occurs in patients with destructive or painless thyroiditis $[26,63]$.

\section{Recommendations}

15. We recommend evaluating patients with newly diagnosed Hyper concerning possible previous radiological examinations using ICM in the last 2-3 months, because it may influence diagnosis (e.g., interpretation of thyroid scintigraphy), prognosis, and treatment. 1, $\varnothing \varnothing \varnothing 0$

16. We recommend that serum TSH should be measured as an initial screening in patients with a suspicion of ICM-induced Hyper. If serum TSH is low, thyroid hormones (T4 and T3) should follow. 1, ØØØØ

17. If the diagnosis of ICM-induced Hyper is not apparent based on the clinical presentation, we suggest analyzing urinary iodine concentration to support iodine excess as the cause of Hyper and to monitor treatment. 2, ØØ०О

18. We recommend determining the etiology of ICM-induced Hyper. Diagnostic testing can include the following: measurement of TSH-receptor antibody, thyroid ultrasound, and/or thyroid scintigraphy. 1, $\varnothing \varnothing 00$ 
What Is the Management of ICM-Induced Hyper?

In the absence of prospective clinical trials on the management of ICM-induced Hyper, an individualized approach to treatment must be advised, based on: (i) the clinical symptoms, etiology, and severity of Hyper and (ii) patients' age, concomitant diseases (especially cardiovascular diseases), and clinical status. The clinical course of ICM-induced Hyper is unpredictable. It is most often mild and self-limited, but occasionally it may lead to clinically significant thyrotoxicosis lasting more than 12 months. Large toxic nodular goiter and Graves' disease may be associated with a more severe course of disease.

Patients with oligo- or asymptomatic SHyper and OHyper should be treated according to local guidelines $[23,25]$. Initial management includes close monitoring and avoidance of further excess iodine exposure. Thyroid function should be retested within 2-8 weeks to determine if the ICM-induced Hyper spontaneously resolves. The symptoms of adrenergic hyperactivity (tachycardia, palpitations, tremor, anxiety, etc.) may be improved through administration of $\beta$-blocking drugs (nonselective or cardio-selective depending on the clinical setting). Severe ICM-induced Hyper is best treated by ATD (methimazole, carbimazole, or propylthiouracil) in addition to $\beta$-blocker. The iodine-loaded thyroid gland may be less responsive to ATD; in such cases, higher doses of ATD (in rare cases up to $40-60 \mathrm{mg} /$ day of methimazole or equivalent doses of propylthiouracil) and/or sodium perchlorate (in rare cases up to 4 weeks course at doses not exceeding $1 \mathrm{~g}$ /day) may be useful in accelerating control of Hyper [26, 63]. Patients who develop severe iodineinduced OHyper should be referred to endocrinologists because the management of this condition can be challenging. Many patients with underlying autonomous nodular thyroid disease are able to taper and discontinue ATD within 6-12 months. Urinary iodine may be monitored to assess the rate of clearance of the iodine load. After resolution of the acute episode of ICM-induced Hyper, treatment of the underlying thyroid disease, if any, should be addressed.

Recommendations

19. We recommend an individualized approach to the treatment of ICM-induced Hyper, based on: (i) clinical symptoms, etiology, and severity of Hyper and (ii) patients' age, concomitant diseases (especially cardiovascular diseases), and clinical status. 1, ØØОО

20. In most mild cases, we recommend close monitoring, avoidance of further excess iodine exposure, and administrating $\beta$-blocking drugs. $1, \varnothing \varnothing \mathrm{OO}$
21. In severe cases, we recommend initiation of treatment with ATD (methimazole, carbimazole, or propylthiouracil). In cases of partial response to ATD, a combination with perchlorate is helpful to control Hyper. 1 $\varnothing 000$

\section{What Are the Recommendations for Geriatric}

\section{Patients?}

ICM-induced Hyper is particularly important in geriatric patients for several reasons:

- The prevalence of thyroid nodular disease increases with age, especially in regions with present or past iodine deficiency. In the elderly population, ICM-induced Hyper may be the first presentation of a previously asymptomatic multinodular goiter.

- Nowadays, elderly patients are more often exposed to radiological studies using ICM than in the past because of major technological advances and increased longevity.

- Hyper in the elderly is more likely to be undiagnosed prior and after ICM-exposure due to the nonspecific nature of the rather few symptoms particularly in the presence of cognitive impairment.

- The number of clinical signs found in the older subjects is significantly fewer than the number found in younger patients. The most common signs associated with thyrotoxicosis in older people include tachycardia, apathy, fatigue, and weight loss $[64,65]$.

- Geriatric patients are less tolerant to the effects of thyrotoxicosis because of underlying heart disease. Moreover, symptoms and signs of heart failure and atrial fibrillation may dominate the clinical picture $[25,57$, $58,66,67]$.

Although randomized clinical trials are pending, in geriatric patients a more aggressive approach pertaining to the prevention of ICM-induced Hyper, early diagnosis and treatment, especially in areas of iodine deficiency is suggested. Furthermore and in accordance with the ETA guidelines, even mild ICM-induced SHyper should be treated in patients over 65 years, particularly in the presence of comorbidity, for example, heart disease, diabetes, renal failure, and previous stroke [25].

\section{Recommendations}

22. We recommend that even mild ICM-induced Hyper should be treated in patients older than 65 years, particularly in the presence of comorbidity. $1, \varnothing \varnothing \varnothing \mathrm{O}$ 
Diagnosis and Management of ICM-Induced Hypo

Establishing the Diagnosis and Etiology of

ICM-Induced Hypo

The diagnosis of ICM-induced Hypo is based upon: (i) the clinical manifestations of Hypo, (ii) laboratory findings, and (iii) history of ICM exposure. The induction of OHypo appears to be rare and the exact timing of the event is unpredictable, ranging from days to up to 2 years after ICM exposure $[13,14,17,18]$. Because of the wide vulnerable time window (from ICM exposure to development of Нypo), general monitoring of thyroid function tests in high-risk patients for Hypo is not advisable. Therefore, all patients should be informed to alert the medical team if signs and symptoms of Hypo occur after ICM administration.

The diagnosis of SHypo and OHypo is confirmed by thyroid function tests (TSH and T4 ). The relationship between Hypo and ICM exposure may be difficult to demonstrate because the urine iodine concentration may be no longer increased.

ICM-induced Hypo is thought to result from a failure to escape the acute Wolff-Chaikoff effect $[3,5,68]$. It is predominantly found in iodine-replete regions. Although ICM-induced Hypo may occur in patients with an apparently normal thyroid gland, most frequently it develops in patients with underlying autoimmune thyroiditis. However, a wide variety of thyroid and nonthyroid diseases were associated with Hypo after ICM exposure (Table 2), and the possible etiology of Hypo should be established [21, 22]. Measurement of serum antithyroid peroxidase antibodies is the most sensitive serological test for the diagnosis of autoimmune thyroiditis.

\section{Recommendations}

23. We recommend that serum TSH should be measured as an initial screening test in patients with a suspicion of ICM-induced Hypo. If serum TSH is high, serum T4 levels should be measured. $1, \varnothing \varnothing \varnothing \varnothing$

24 . We recommend measurement of serum thyroid peroxidase antibodies if the etiology of ICM-induced Hypo is unclear. $1, \varnothing \varnothing 00$

25. We suggest evaluating patients with newly diagnosed Hypo concerning previous radiological examinations using ICM (in the last 1-2 years), since spontaneous remission might occur over time, without the need for lifelong temporary levothyroxine (LT4) treatment. 2, $\varnothing 000$
What Is the Management of ICM-Induced Hypo?

There are no prospective clinical trials on the management of ICM-induced Hypo, which is usually self-limiting $[56,68]$. However, the time to recovery may vary from several weeks up to several months. Prolonged ICM-induced Hypo may be observed in patients, in whom iodine excess is not rapidly eliminated from the body (e.g. repeated exposure to excess iodine, the use of oil-soluble ICM, and renal failure). In rare cases, ICM-induced Hypo may be permanent.

Most patients do not require thyroid hormone replacement and TSH should be retested after 4-8 weeks. LT4 treatment may be considered based on individual patient factors, including: younger age, symptoms of Hypo, presence of an autoimmune thyroid disease, and comorbidities. ICM-induced Hypo in pregnant women or women planning pregnancy should be treated as all other forms of Hypo [21]. After a few months, LT4 treatment may be tapered to determine if ICM-induced Hypo has resolved. However, patients with underlying thyroid disease, who develop transient ICM-induced Hypo, are at risk for permanent Hypo in the future.

\section{Recommendations}

26. We recommend an individualized approach to the treatment of ICM-induced Hypo, based on: (i) clinical symptoms, etiology and severity of Hypo, and (ii) patients' age, concomitant diseases, and clinical status. 1 , $\varnothing \varnothing О О$

27. In most cases of ICM-induced Hypo, we suggest close monitoring without thyroid hormone replacement. Temporary LT4 treatment may be considered in younger patients with symptoms of Hypo, with an underlying chronic autoimmune thyroiditis, and in women planning pregnancy. 2, ØØОО

What Are the Recommendations for the Elderly?

Old age was shown to be correlated with the risk of ICM-induced Hypo. High-risk elderly patients for ICMinduced Hypo should be carefully evaluated after radiological examination with ICM because OHypo in these patients is usually oligosymptomatic. Moreover, most common symptoms are nonspecific (e.g. tiredness, shortness of breath, depression, and cognitive changes), while the more classical Hypo-associated symptoms may be absent $[69,70]$. On the other hand, elderly hypothyroid patients are also more sensitive to exogenous thyroid hormones. If needed, LT4 replacement should be started at lower doses and titrated as per response to avoid overtreatment $[21,22,26]$. 
Table 3. Summary of recommendations

Question/topic Recommendation (strength of and level of evidence)

Recommendations before radiological examinations using ICM

Screening of baseline thyroid function 1 . We do not recommend baseline thyroid function testing of the general population before prior to ICM exposure in the general radiological examinations using ICM administration. 1, ØØOO

population

Screening of baseline thyroid function in high-risk patients for ICM-induced TD

2. Prior to ICM exposure, we suggest a thorough case-finding approach, based on identifying those persons most likely to have undiagnosed TD. 2, ØØОО

3. We suggest measurement of baseline serum TSH in high-risk patients for ICM-induced $\mathrm{TD}$, especially in the elderly and subjects at risk for cardiovascular diseases. If serum TSH is abnormal, thyroid hormones (T3 and/or T4) should be measured. 2, ØОOО

Is baseline TD a contraindication to ICM administration?

4. We do not recommend radiological examinations using ICM in patients with OHyper. Alternative imaging procedures (e.g., ultrasound, MRI, and noncontrast CT) should be considered. 1, ØØОО

5. Persistent endogenous SHyper is not a contraindication to radiological examinations using ICM. Prior to elective radiological studies, we recommend to establish the etiology of SHyper. Endocrine consultation may be advised. 1, ØØØО

6. Baseline untreated SHypo and OHypo are not a contraindication to ICM administration. Due to possible exacerbation of Hypo, close monitoring after ICM is suggested in selected patients. 2, ØØОО

7. Patients on thyroid hormone replacement are not at risk for ICM-induced TD and do not require specific management. $1, \varnothing \mathrm{OOO}$

Should high-risk patients for ICMinduced Hyper receive prophylactic treatment before a radiological examination?
8. We do not recommend prophylactic treatment in all patients at high-risk for developing ICM-induced Hyper. However, patients at risk for Hyper should be monitored after ICM injection. 1, ØØОО

9. We suggest considering prophylactic treatment with methimazole and/or perchlorate before emergency radiological examinations in selected patients who are less tolerant to the effects of ICM-induced Hyper, for example, elderly with persistent endogenous SHyper and/ or nodular goiter, and/or concomitant cardiovascular disease, especially in areas of iodine deficiency. 2, ØØОО

Monitoring of patients after radiological examinations using ICM

Does ICM exposure affect thyroid function tests in euthyroid subjects? after ICM administration?
Should thyroid function be monitored

10. ICM administration may cause transient changes in serum TSH, T4, and T3 levels in healthy individuals, usually within the reference values. While interpreting thyroid function tests, we suggest taking ICM exposure (within the last 1-3 months) into consideration. 2, $\varnothing \varnothing \varnothing 0$

11. We do not recommend routine TSH monitoring following ICM administration. 1, $\varnothing \varnothing \varnothing О$

12. The exact timing of the ICM-TD is often unpredictable. We suggest advising patients to alert the medical team if they develop signs and symptoms of TD. 2, ØOOO

13. We suggest measuring thyroid function 3-4 weeks after ICM exposure in high-risk patients for ICM-induced Hyper, especially in the elderly and/or those with an underlying unstable cardiovascular disease. 2, ØØ०О

When can isotope imaging of the thyroid or RAI treatment be performed after ICM exposure?
14. We recommend avoiding isotope imaging of the thyroid and radioactive iodine treatment for one to 2 months after ICM injection. $1, \varnothing \varnothing \varnothing \mathrm{O}$ 
Table 3 (continued)

Question/topic

Recommendation (strength of and level of evidence)

Diagnosis and management of ICM-induced Hyper

Establishing the diagnosis and etiology 15 . We recommend evaluating patients with newly diagnosed Hyper concerning possible of ICM-induced Hyper previous radiological examinations using ICM in the last 2-3 months because it may influence diagnosis (e.g., interpretation of thyroid scintigraphy), prognosis, and treatment. 1, $\varnothing \varnothing \varnothing О$

16. We recommend that serum TSH should be measured as an initial screening in patients with a suspicion of ICM-induced Hyper. If serum TSH is low, thyroid hormones (T4 and T3) should follow. $1, \varnothing \varnothing \varnothing \varnothing$

17. If the diagnosis of ICM-induced Hyper is not apparent based on the clinical presentation, we suggest analyzing urinary iodine concentration to support iodine excess as the cause of Hyper and to monitor treatment. 2, ØØОО

18. We recommend determining the etiology of ICM-induced Hyper. Diagnostic testing can include the following: measurement of TSHR-Ab, thyroid ultrasound, and/or thyroid scintigraphy. $1, \varnothing \varnothing \mathrm{OO}$

What is the management of ICM-

19. We recommend an individualized approach to the treatment of ICM-induced Hyper, induced Hyper? based on: (i) clinical symptoms, etiology and severity of Hyper and (ii) patients' age, concomitant diseases (especially cardiovascular diseases), and clinical status. $1, \varnothing \varnothing \mathrm{\text {, }}$

20. In most mild cases, we recommend close monitoring, avoidance of further excess iodine exposure, and administrating $\beta$-blocking drugs $1, \varnothing \varnothing 0 \bigcirc$

21. In severe cases, we recommend initiation of treatment with ATD (methimazole, carbimazole, or propylthiouracil). In cases of partial response to ATD, a combination with perchlorate is helpful to control Hyper. $1 \varnothing 00 \mathrm{O}$

What are the recommendations for the 22. We recommend that even mild ICM-induced Hyper should be treated in patients older elderly? than 65 years, particularly in the presence of comorbidity. $1, \varnothing \varnothing \varnothing 0$

Diagnosis and management of ICM-induced Hypo

Establishing the diagnosis and etiology of ICM-induced Hypo
23. We recommend that serum TSH should be measured as an initial screening test in patients with a suspicion of ICM-induced Hypo. If serum TSH is high, serum T4 levels should be measured. $1, \varnothing \varnothing \varnothing \varnothing$

24. We recommend measurement of serum TPO-Ab if the etiology of ICM-induced Hypo is unclear. $1, \varnothing \varnothing 00$

25. We suggest evaluating patients with newly diagnosed Hypo concerning previous radiological examinations using ICM (in the last $1-2$ years) since spontaneous remission might occur over time, without the need for lifelong LT4 treatment. 2, ØООО

What is the management of ICMinduced Hypo?

26. We recommend an individualized approach to the treatment of ICM-induced Hypo, based on: (i) Clinical symptoms, etiology, and severity of Hypo and (ii) patients' age, concomitant diseases, and clinical status. $1, \varnothing \varnothing \bigcirc$

27. In most cases of ICM-induced Hypo, we suggest close monitoring without thyroid hormone replacement. LT4 treatment may be considered in younger patients with symptoms of Hypo, with an underlying chronic autoimmune thyroiditis and in women planning pregnancy. $2, \varnothing \varnothing \mathrm{OO}$

What are the recommendations for the 28. Elderly patients with ICM-induced SHypo should be carefully followed, generally elderly? avoiding levothyroxine treatment. 2, ØØОО

What are the recommendations for infants?

29 . We suggest careful monitoring of thyroid function tests in infants after maternal or neonatal ICM exposure, with a special attention to premature infants. $2, \varnothing \varnothing \mathrm{OO}$

ICM, iodine-based contrast media; TD, thyroid dysfunction; Hyper, hyperthyroidism; Hypo, hypothyroidism; CT, computed tomography; SHyper, subclinical Hyper; SHypo, subclinical Hypo; ATD, antithyroid drug; RAI, radioiodine; TSHR-Ab, TSH-receptor antibody; TPO-Ab, thyroid peroxidase antibodies; LT4, levothyroxine. 
Recommendations

28. Elderly patients with ICM-induced SHypo should be carefully followed, generally avoiding levothyroxine treatment. 2, ØØ००

What Are the Recommendations for Infants?

Premature infants and neonates might be particularly susceptible to the development of ICM-induced Hypo because the immature gland may not be able to fully escape from the acute Wolff-Chaikoff effect [49]. In 2015, the US Food and Drug Administration issued a safety announcement regarding 10 cases of ICM-induced Hypo in infants younger than 4 months, reported between 1969 and 2012 . In all cases, the infants were either premature or had serious cardiac abnormalities. Hypo occurred within 1 month after receiving ICM. Four of the 10 infants received LT4 treatment for up to 10 months, while others improved without treatment. Based on these observations, healthcare professionals were advised to use clinical judgment to determine whether testing for Hypo might be necessary after ICM exposure (https://www.fda.gov/drugs/ drug-safety-and-availability/fda-drug-safety-communication-fda-advises-rare-cases-underactive-thyroid-infants-given-iodine.)

However, prevalence, severity, and duration of ICM-induced Hypo in neonates remain largely unknown. A systemic review, including eleven studies (182 neonates) attempted to determine the risk of Hypo after ICM exposure, found inconsistent results for term infants (one study did not show an increased risk, while another did find a trend towards increased TSH levels and decreased FT4 levels) [71]. In contrast, studies including premature infants found trends toward increased serum TSH, decreased T3 and FT4, and the need for LT4 treatment $(8.3 \%$ of the term infants and $18.3 \%$ of the premature infants were treated for ICMinduced Hypo). Unfortunately, all studies included in the systemic review were highly affected by bias [71]. In contrast, mild, transient, neonatal Hypo was seen following exposure to iodine via caesarean section but not via exposure to contrast media [72]. Only one prospective randomized study has compared thyroid function tests in preterm neonates exposed to ICM with controls after peripherally inserted central catheter (PICC) at day 14 post PICC insertion or on day 28 of life. The results suggested that $0.3 \mathrm{~mL}$ of iodine-containing contrast material could be administered to extreme preterm neonates for better PICC tip visualization without causing Hypo [73].

The most frequently reported risk factors for ICM-induced Hypo (especially in preterm neonates) are: underlying congenital cardiac malformations, impaired renal function, and multiple radiological examinations [74]. Clinically, significant OHypo may occur after various radiological examinations using ICM [75-78]. In addition, there have been cases of transient fetal goiter or transient neonatal Hypo due to preconception or postnatal maternal exposure to ICM $[79,80]$. However, a recent retrospective study suggested that preconception hysterosalpingography did not influence the neonatal thyroid function [50].

Although ICM-induced Hypo is likely transient and of short duration, its effects on the developing neonatal brain may be harmful. Until more evidence is available, we suggest careful monitoring of thyroid function tests in infants after maternal or neonatal ICM exposure, with a special attention to premature infants. ICM-induced Hypo in newborns should be treated according to local guidelines [81].

\section{Recommendations}

29. We suggest careful monitoring of thyroid function tests in infants after maternal or neonatal ICM exposure, with a special attention to premature infants. 2 , $\varnothing \varnothing 00$

\section{Conclusions}

Evidence in the field of ICM-induced TD is scarce, resulting in the majority of cases in weak recommendations predominantly based on low or very low-quality evidence, which are summarized in Table 3. Although most cases of ICM-induced Hyper are mild and transient, there is a small risk of severe thyrotoxicosis with serious cardiovascular complications, especially in elderly patients. On the other hand, the fetus and neonate may be susceptible to developing clinically significant Hypo after maternal or neonatal ICM exposure. An individualized approach to prevention and treatment of ICM-induced TD is advised, based on patient's age, clinical symptoms of TD, pre-existing thyroid diseases, coexisting morbidities, and iodine intake. Additional clinical trials with clinically relevant endpoints are warranted to further aid in clinical decision-making in patients with ICM-induced TD.

\section{Acknowledgements}

The task force wishes to thank the ETA Guidelines and Publications Board for their constant help and support, as well as ETA members (Professor Paolo Beck-Peccoz, Professor Bekir Cakir, Dr Oana Capraru, Professor Alicja Hubalewska-Dydejczyk, and Pro- 
fessor Anders Karlsson) for their thoughtful suggestions. The authors would also like to thank Dr Justyna Milczarek-Banach for her help in editing the reference list.

\section{Conflict of Interest Statement}

T.B. has received speaker honoraria from Berlin-Chemie/Menarini, Ipsen, Merck, and Novartis. He has been a consultant for Ipsen and Merck. T.H.B. has no conflicts of interest to declare. W.S. has received speaker honoraria from Siemens Healthineers and GE Healthcare. G.Z. is shareholder of Piur Imaging GmbH and $\mathrm{ZM}$ Sono $\mathrm{GmbH}$ and has received speaker honoraria from Genericon Pharma. The Johannes Gutenberg University (JGU) Medical Center, Mainz, Germany, academic institution of G.J.K. has received research-associated funding from the JGU Medical Faculty, AdvanceCor (Germany), Apitope (Belgium), Horizon (USA), Immunovant (USA), ISAR (Germany), Mediomics (USA),
Merck (Germany), Novartis (USA), Quidel (USA), River Vision (USA), Roche (Switzerland), and Byondis (The Netherlands). G.J.K. consults for Immunovant, Mediomics, Merck, Novartis, and Quidel.

\section{Funding Sources}

No funding was received to support this work.

\section{Author Contributions}

All the authors contributed to the idea, gathered the information, interpreted the data, and wrote and accepted the final version of the manuscript.

\section{References}

1 Persson PB. Editorial: contrast medium-induced nephropathy. Nephrol Dial Transplant. 2005 Feb;20(Suppl 1):i1.

2 van der Molen AJ, Thomsen HS, Morcos SK; Contrast Media Safety Committee ErSoURE. Effect of iodinated contrast media on thyroid function in adults. Eur Radiol. 2004 May; 14(5):902-7.

3 Leung AM, Braverman LE. Iodine-induced thyroid dysfunction. Curr Opin Endocrinol Diabetes Obes. 2012 Oct;19(5):414-9.

4 Hudzik B, Zubelewicz-Szkodzińska B. Radiocontrast-induced thyroid dysfunction: is it common and what should we do about it? Clin Endocrinol (Oxf). 2014 Mar;80(3):3227.

5 Lee SY, Rhee CM, Leung AM, Braverman LE, Brent GA, Pearce EN. A review: radiographic iodinated contrast media-induced thyroid dysfunction. J Clin Endocrinol Metab. 2015 Feb;100(2):376-83.

6 Thomsen HS, Bellin MF, Jakobsen JA, Webb JAW. Contrast media classification and terminology. In: Thomsen HS, Webb JAW, editors. Contrast media safety issues and ESUR guideline. Heidelberg: Springer; 2014. p. 3-11

7 Spampinato MV, Abid A, Matheus MG. Current radiographic iodinated contrast agents. Magn Reson Imaging Clin N Am. 2017 Nov; 25(4):697-704.

8 Dawson P, Crosgrove DO, Grainger RG. Textbook of contrast media. Oxford: ISIS Medical Media; 1999.

9 Solomon R. Contrast media: are there differences in nephrotoxicity among contrast media? Biomed Res Int. 2014;2014:934947.

10 Lell MM, Jost G, Korporaal JG, Mahnken AH, Flohr TG, Uder M, et al. Optimizing contrast media injection protocols in state-of-the art computed tomographic angiography. Invest Radiol. 2015 Mar;50(3):161-7.
11 Jensen CT, Wagner-Bartak NA, Vu LN, Liu X, Raval B, Martinez D, et al. Detection of colorectal hepatic metastases is superior at standard radiation dose CT versus reduced dose CT. Radiology. 2019 Feb;290(2):400-9.

12 Martens B, Hendriks BMF, Eijsvoogel NG, Wildberger JE, Mihl C. Individually body weight-adapted contrast media application in computed tomography imaging of the liver at 90 kVp. Invest Radiol. 2019 Mar;54(3):17782.

13 Rhee CM, Bhan I, Alexander EK, Brunelli SM. Association between iodinated contrast media exposure and incident hyperthyroidism and hypothyroidism. Arch Intern Med. 2012 Jan;172(2):153-9.

14 Kornelius E, Chiou JY, Yang YS, Peng CH, Lai YR, Huang CN. Iodinated contrast media increased the risk of thyroid dysfunction: a 6-year retrospective cohort study. J Clin Endocrinol Metab. 2015 Sep;100(9): 3372-9.

15 Hsieh MS, Chiu CS, Chen WC, Chiang JH, Lin SY, Lin MY, et al. Iodinated contrast medium exposure during computed tomography increase the risk of subsequent development of thyroid disorders in patients without known thyroid disease: a nationwide population-based, propensity score-matched, longitudinal follow-up study. Medicine. 2015 Dec; 94(50):e2279.

16 Kornelius E, Chiou JY, Yang YS, Lo SC, Peng $\mathrm{CH}$, Lai YR, et al. Iodinated contrast mediainduced thyroid dysfunction in euthyroid nodular goiter patients. Thyroid. 2016 Aug; 26(8):1030-8.

17 Jick SS, Hedderson M, Xu F, Cheng Y, Palkowitsch P, Michel A. Iodinated contrast agents and risk of hypothyroidism in young children in the United States. Invest Radiol. 2019 May;54(5):296-301.
18 Barr ML, Chiu HK, Li N, Yeh MW, Rhee CM, Casillas J, et al. Thyroid dysfunction in children exposed to iodinated contrast media. J Clin Endocrinol Metab. 2016;101(6):236670.

19 Bervini S, Trelle S, Kopp PA, Stettler C, Trepp R. Incidence of iodine-induced hyperthyroidism after administration of iodinated contrast during radiographic procedures: a systematic review and meta-analysis of the literature. Thyroid. 2021 Mar 15. Epub ahead of print.

20 Thomsen HS. European Society of Urogenital Radiology (ESUR) guidelines on the safe use of iodinated contrast media. Eur J Radiol. 2006 Dec;60(3):307-13.

21 Garber JR, Cobin RH, Gharib H, Hennessey JV, Klein I, Mechanick JI, et al. Clinical practice guidelines for hypothyroidism in adults: cosponsored by the American Association of Clinical Endocrinologists and the American Thyroid Association. Endocr Pract. 2012 Nov-Dec;18(6):988-1028.

22 Pearce SH, Brabant G, Duntas LH, Monzani F, Peeters RP, Razvi S, et al. ETA guideline: management of subclinical hypothyroidism. Eur Thyroid J. 2013 Dec;2(4):215-28.

23 Ross DS, Burch HB, Cooper DS, Greenlee MC, Laurberg P, Maia AL, et al. 2016 American thyroid association guidelines for diagnosis and management of hyperthyroidism and other causes of thyrotoxicosis. Thyroid. 2016; 26(10):1343-421.

24 Rusandu A, Siøvold BH, Hofstad E, Reidunsdatter RJ. Iodinated contrast media and their effect on thyroid function - routines and practices among diagnostic imaging departments in Norway. J Med Radiat Sci. 2020 Jun; 67(2):111-8 
25 Biondi B, Bartalena L, Cooper DS, Hegedüs L, Laurberg P, Kahaly GJ. The 2015 European thyroid association guidelines on diagnosis and treatment of endogenous subclinical hyperthyroidism. Eur Thyroid J. 2015 Sep;4(3): 149-63.

26 Bartalena L, Bogazzi F, Chiovato L, Hubalewska-Dydejczyk A, Links TP, Vanderpump M. European Thyroid Association (ETA) guidelines for the management of amiodarone-associated thyroid dysfunction. Eur Thyroid J. 2018 Mar;7(2):55-66.

27 Kahaly GJ, Bartalena L, Hegedüs L, Leenhardt L, Poppe K, Pearce SH. 2018 European thyroid association guideline for the management of Graves' hyperthyroidism. Eur Thyroid J. 2018 Aug;7(4):167-86.

28 de Bruin TW. Iodide-induced hyperthyroidism with computed tomography contrast fluids. Lancet. 1994 May;343(8906):1160-1.

29 Thomsen HS, Faber J. Iodine-based contrast media cause hyperthyroidism - another important adverse reaction to contrast media? Acta Radiol. 2012 Dec;53(10):1079-80.

30 Bonelli N, Rossetto R, Castagno D, Anselmino M, Vignolo F, Parasiliti Caprino M, et al. Hyperthyroidism in patients with ischaemic heart disease after iodine load induced by coronary angiography: long-term follow-up and influence of baseline thyroid functional status. Clin Endocrinol. 2018 Feb;88(2):272-8.

31 Marraccini P, Bianchi M, Bottoni A, Mazzarisi A, Coceani M, Molinaro S, et al. Prevalence of thyroid dysfunction and effect of contrast medium on thyroid metabolism in cardiac patients undergoing coronary angiography. Acta Radiol. 2013 Feb;54(1): $42-7$.

32 Phillips BD, Hennessey JV. Iodinated contrast prior to evaluation for thyrotoxicosis. J Hosp Med. 2009 May;4(5):285-8.

33 Hennessey JV, Garber JR, Woeber KA, Cobin R, Klein I, Committee ATS, et al. American association of clinical endocrinologists and American college of endocrinology position statement on thyroid dysfunction case finding. Endocr Pract. 2016 Feb;22(2):262-70.

34 Pelewicz K, Wolny R, Bednarczuk T, Miśkiewicz P. Prevention of iodinated contrast media-induced hyperthyroidism in patients with euthyroid goiter. Eur Thyroid J. 2021.

35 Treglia G, Trimboli P, Verburg FA, Luster M, Giovanella L. Prevalence of normal TSH value among patients with autonomously functioning thyroid nodule. Eur J Clin Invest. $2015 \mathrm{Jul}$ 45(7):739-44.

36 Bish LT, Bavaria JE, Augoustides J. Thyroid storm after coronary artery bypass grafting. J Thorac Cardiovasc Surg. 2010 Nov; 140(5): e67-9.

37 Alkhuja S, Pyram R, Odeyemi O. In the eye of the storm: iodinated contrast medium induced thyroid storm presenting as cardiopulmonary arrest. Heart Lung. 2013 Jul-Aug; 42(4):267-9.
38 Brundridge W, Perkins J. Iodinated contrast administration resulting in cardiogenic shock in patient with uncontrolled graves disease. J Emerg Med. 2017 Dec;53(6):e125-28.

39 Fricke E, Fricke H, Esdorn E, Kammeier A, Lindner O, Kleesiek K, et al. Scintigraphy for risk stratification of iodine-induced thyrotoxicosis in patients receiving contrast agent for coronary angiography: a prospective study of patients with low thyrotropin. J Clin Endocrinol Metab. 2004 Dec;89(12):6092-6.

40 Fritzsche H, Benzer W, Furlan W, Hammerle D, Langsteger W, Weiss P. [Prevention of iodine-induced hyperthyroidism after coronary angiography]. Acta Med Austriaca. 1993; 20(1-2):13-7.

41 Schaffhauser K, Hänscheid H, Rendl J, Grelle I, Reiners C. [Intrathyroidal iodine concentration after application of non-ionic contrast media with and without prophylactic application of perchlorate]. Nuklearmedizin. 2005; 44(4):143-8.

42 Nolte, Muller R, Siggelkow H, Emrich D, Hufner M. Prophylactic application of thyrostatic drugs during excessive iodine exposure in euthyroid patients with thyroid autonomy: a randomized study. Eur J Endocrinol. 1996 Mar;134(3):337-41.

43 Available from: https://www.schilddruesengesellschaft.at/.

44 Koroscil TM, Pelletier PR, Slauson JW, Hennessey J. Short-term effects of coronary angiographic contrast agents on thyroid function. Endocr Pract. 1997 Jul-Aug;3(4):219-21.

45 Gartner W, Weissel M. Do iodine-containing contrast media induce clinically relevant changes in thyroid function parameters of euthyroid patients within the first week? Thyroid. 2004 Jul;14(7):521-4.

46 Lee SY, Chang DL, He X, Pearce EN, Braverman LE, Leung AM. Urinary iodine excretion and serum thyroid function in adults after iodinated contrast administration. Thyroid. 2015 May;25(5):471-7.

47 Bøhmer T, Bachtyari Z, Sommer C, Hammerstad SS. Auto regulatory capacity of the thyroid gland after numerous iodinated contrast media investigations. Scand J Clin Lab Invest. 2020 May;80(3):191-5.

48 Kaneshige T, Arata N, Harada S, Ohashi T, Sato S, Umehara N, et al. Changes in serum iodine concentration, urinary iodine excretion and thyroid function after hysterosalpingography using an oil-soluble iodinated contrast medium (lipiodol). J Clin Endocrinol Metab. 2015 Mar;100(3):E469-72.

49 Maloney E, Iyer RS, Phillips GS, Menon S, Lee JJ, Callahan MJ. Practical administration of intravenous contrast media in children: screening, prophylaxis, administration and treatment of adverse reactions. Pediatr Radiol. 2019 Apr;49(4):433-47.

50 van Welie N, Roest I, Portela M, van Rijswijk J, Koks C, Lambalk CB, et al. Thyroid function in neonates conceived after hysterosalpingography with iodinated contrast. Hum Reprod. 2020 May;35(5):1159-67.
51 Nygaard B, Nygaard T, Jensen LI, Court-Payen M, Søe-Jensen P, Nielsen KG, et al. Iohexol: effects on uptake of radioactive iodine in the thyroid and on thyroid function. Acad Radiol. 1998 Jun;5(6):409-14.

52 Nimmons GL, Funk GF, Graham MM, Pagedar NA. Urinary iodine excretion after contrast computed tomography scan: implications for radioactive iodine use. JAMA Otolaryngol Head Neck Surg. 2013 May;139(5): 479-82.

53 Padovani RP, Kasamatsu TS, Nakabashi CC, Camacho CP, Andreoni DM, MaloufEZ, et al. One month is sufficient for urinary iodine to return to its baseline value after the use of water-soluble iodinated contrast agents in postthyroidectomy patients requiring radioiodine therapy. Thyroid. 2012 Sep;22(9):926-30.

54 Haugen BR, Alexander EK, Bible KC, Doherty GM, Mandel SJ, Nikiforov YE, et al. 2015 American thyroid association management guidelines for adult patients with thyroid nodules and differentiated thyroid cancer: the American thyroid association guidelines task force on thyroid nodules and differentiated thyroid cancer. Thyroid. 2016 Jan;26(1):1133.

55 Fassbender WJ, Vogel C, Doppl W, Stracke H, Bretzel RG, Klör HU. Thyroid function, thyroid immunoglobulin status, and urinary iodine excretion after enteral contrast-agent administration by endoscopic retrograde cholangiopancreatography. Endoscopy. 2001 Mar;33(3):245-52.

56 Mekaru K, Kamiyama S, Masamoto H, Sakumoto K, Aoki Y. Thyroid function after hysterosalpingography using an oil-soluble iodinated contrast medium. Gynecol Endocrinol. 2008 Sep;24(9):498-501.

57 Kahaly GJ, Dillmann WH. Thyroid hormone action in the heart. Endocr Rev. 2005 Aug; 26(5):704-28.

58 Biondi B, Kahaly GJ. Cardiovascular involvement in patients with different causes of hyperthyroidism. Nat Rev Endocrinol. 2010 Aug;6(8):431-43.

59 Lillevang-Johansen M, Abrahamsen B, Jørgensen HL, Brix TH, Hegedüs L. Excess mortality in treated and untreated hyperthyroidism is related to cumulative periods of low serum TSH. J Clin Endocrinol Metab. 2017 Jul;102(7):2301-9.

60 Hintze G, Blombach O, Fink H, Burkhardt U, Köbberling J. Risk of iodine-induced thyrotoxicosis after coronary angiography: an investigation in 788 unselected subjects. Eur J Endocrinol. 1999 Mar;140(3):264-7.

61 Pedersen IB, Knudsen N, Perrild H, Ovesen L, Laurberg P. TSH-receptor antibody measurement for differentiation of hyperthyroidism into Graves' disease and multinodular toxic goitre: a comparison of two competitive binding assays. Clin Endocrinol. 2001 Sep;55(3): 381-90.

62 Kahaly GJ, Diana T, Olivo PD. TSH receptor antibodies: relevance \& utility. Endocr Pract. 2020 Jan;26(1):97-106. 
63 Jabrocka-Hybel A, Bednarczuk T, Bartalena L, Pach D, Ruchała M, Kamiński G, et al. Amiodarone and the thyroid. Endokrynol Pol. 2015;66(2):176-86.

64 Trivalle C, Doucet J, Chassagne P, Landrin I, Kadri N, Menard JF, et al. Differences in the signs and symptoms of hyperthyroidism in older and younger patients. J Am Geriatr Soc. 1996 Jan;44(1):50-3.

65 Boelaert K, Torlinska B, Holder RL, Franklyn JA. Older subjects with hyperthyroidism present with a paucity of symptoms and signs: a large cross-sectional study. J Clin Endocrinol Metab. 2010 Jun;95(6):2715-26.

66 Martin FI, Tress BW, Colman PG, Deam DR. Iodine-induced hyperthyroidism due to nonionic contrast radiography in the elderly. Am J Med. 1993 Jul;95(1):78-82.

67 Martin FI, Deam DR. Hyperthyroidism in elderly hospitalised patients. Clinical features and treatment outcomes. Med J Aust. 1996 Feb;164(4):200-3.

68 Markou K, Georgopoulos N, Kyriazopoulou $\mathrm{V}$, Vagenakis AG. Iodine-induced hypothyroidism. Thyroid. 2001 May;11(5):501-10.

69 Davis JD, Tremont G. Neuropsychiatric aspects of hypothyroidism and treatment reversibility. Minerva Endocrinol. 2007 Mar; 32(1):49-65.

70 Carlé A, Pedersen IB, Knudsen N, Perrild H, Ovesen L, Andersen S, et al. Hypothyroid symptoms fail to predict thyroid insufficiency in old people: a population-based case-control study. Am J Med. 2016 Oct;129(10): 1082-92.

71 Ahmet A, Lawson ML, Babyn P, Tricco AC. Hypothyroidism in neonates post-iodinated contrast media: a systematic review. Acta Paediatr. 2009 Oct;98(10):1568-74.

72 Williams FL, Watson J, Day C, Soe A, Somisetty SK, Jackson L, et al. Thyroid dysfunction in preterm neonates exposed to iodine. J Perinat Med. 2017 Jan;45(1):135-43.

73 Rath CP, Thomas M, Sullivan D, Kluckow M. Does the use of an iodine-containing contrast agent to visualise the PICC tip in preterm babies cause hypothyroidism? A randomised controlled trial. Arch Dis Child Fetal Neonatal Ed. 2019 Mar;104(2):F212-F14.

74 Heo YJ, Lee YA, Lee B, Lee YJ, Lim YH, Chung HR, et al. How can the occurrence of delayed elevation of thyroid stimulating hormone in preterm infants born between 35 and 36 weeks gestation be predicted? PLoS One. 2019;14(8):e0220240.

75 Thaker VV, Leung AM, Braverman LE, Brown RS, Levine B. Iodine-induced hypothyroidism in full-term infants with congenital heart disease: more common than currently appreciated? J Clin Endocrinol Metab. 2014 Oct;99(10):3521-6.
76 Thaker VV, Galler MF, Marshall AC, Almodovar MC, Hsu HW, Addis CJ, et al. Hypothyroidism in infants with congenital heart disease exposed to excess iodine. J Endocr Soc. 2017 Aug;1(8):1067-78.

77 Cherella CE, Breault DT, Thaker V, Levine BS, Smith JR. Early identification of primary hypothyroidism in neonates exposed to intralymphatic iodinated contrast: a case series. J Clin Endocrinol Metab. 2018 Oct;103(10): 3585-8.

78 Cohen L, Pouletty M, Frerot A, Tanase A, Ali L, Baudouin V. Voiding cystography: an unusual route of induced hypothyroidism by iodine overdose in two newborns with chronic kidney disease. Pediatr Nephrol. 2019 Jul; 34(7):1295-7.

79 Omoto A, Kurimoto C, Minagawa M, Shozu M. A case of fetal goiter that resolved spontaneously after birth. J Clin Endocrinol Metab. 2013 Oct;98(10):3910-1.

80 Themelin C, Pierron C, Calafat JF, de Beaufort C. Transient neonatal hypothyroidism secondary to postnatal maternal exposure to contrast medium. BMJ Case Rep. 2019 Oct; 12(10):e230854.

81 Léger J, Olivieri A, Donaldson M, Torresani T, Krude H, van Vliet G, et al. European Society for Paediatric Endocrinology consensus guidelines on screening, diagnosis, and management of congenital hypothyroidism. Horm Res Paediatr. 2014 Feb;81(2):80-103.

82 Ittermann T, Albrecht D, Arohonka P, Bilek R, de Castro JJ, Dahl L, et al. Standardized map of iodine status in Europe. Thyroid. 2020;30(9):1346-54.

83 Bajuk V, Zaletel K, Pirnat E, Hojker S, Gaberšček S. Effects of adequate iodine supply on the incidence of iodine-induced thyroid disorders in Slovenia. Thyroid. $2017 \mathrm{Apr}$ 27(4):558-66

84 Roti E, Minelli R, Gardini E, Bianconi L, Neri $\mathrm{T}$, Gavaruzzi G, et al. Impaired intrathyroidal iodine organification and iodine-induced hypothyroidism in euthyroid women with a previous episode of postpartum thyroiditis. J Clin Endocrinol Metab. 1991 Nov;73(5):958-63.

85 Clark OH, Cavalieri RR, Moser C, Ingbar SH Iodide-induced hypothyroidism in patients after thyroid resection. Eur J Clin Invest. 1990 Dec;20(6):573-80.

86 Alexandrides T, Georgopoulos N, Yarmenitis S, Vagenakis AG. Increased sensitivity to the inhibitory effect of excess iodide on thyroid function in patients with beta-thalassemia major and iron overload and the subsequent development of hypothyroidism. Eur J Endocrinol. 2000 Sep;143(3):319-25.
87 Rhee CM. Thyroid disease in end-stage renal disease. Curr Opin Nephrol Hypertens. 2019 Nov;28(6):621-30.

88 Özkan S, Oysu AS, Kayataş K, Demirtunç R, Eren M, Uslu H, et al. Thyroid functions after contrast agent administration for coronary angiography: a prospective observational study in euthyroid patients. Anadolu Kardiyol Derg. 2013 Jun;13(4):363-9.

89 Skórkowska-Telichowska K, Kosińska J, Szymczak R, Tuchendler D, Tuchendler R, Telichowski A, et al. Comparison and assessment of thyroid morphology and function in inhabitants of Lower Silesia before and after administration of a single dose of iodine-containing contrast agent during cardiac intervention procedure. Endokrynol Pol. 2012; 63(4):294-9.

90 Jarvis C, Simcox K, Tamatea JA, McAnulty K, Meyer-Rochow GY, Conaglen JV, et al. A low incidence of iodine-induced hyperthyroidism following administration of iodinated contrast in an iodine-deficient region. Clin Endocrinol. 2016 Apr;84(4):558-63.

91 Conn JJ, Sebastian MJ, Deam D, Tam M, Martin FI. A prospective study of the effect of nonionic contrast media on thyroid function. Thyroid. 1996 Apr;6(2):107-10.

92 Ledingham D, Carey P, Junejo S. The dangers of iodine-based contrasts in an elderly patient with thyroid disease. BMJ Case Rep. 2015 Mar.

93 Iakovou I, Zapandiotis A, Mpalaris V, Goulis DG. Radio-contrast agent-induced hyperthyroidism: case report and review of the literature. Arch Endocrinol Metab. 2016 Feb;60(3): 287-9.

94 Ma G, Mao R, Zhai H. Hyperthyroidism secondary to hysterosalpingography: an extremely rare complication: a case report. Medicine. 2016 Dec;95(49):e5588.

95 Dunne P, Kaimal N, MacDonald J, Syed AA. Iodinated contrast-induced thyrotoxicosis. CMAJ. 2013 Feb;185(2):144-7.

96 Mushtaq U, Price T, Laddipeerla N, Townsend A, Broadbridge V. Contrast induced hyperthyroidism due to iodine excess. BMJ Case Rep. 2009;2009.

97 Dave A, Ludlow J, Malaty J. Thyrotoxicosis: an under-recognised aetiology. BMJ Case Rep. May 2015;2015:bcr2014208119.

98 Arlt S, Burkhardt D, Wiedemann K. Thyrotoxicosis after iodine contrast medium administration: rapid mood swing to mania and subsequent psychotic depression in a patient with bipolar disorder during lithium therapy. Pharmacopsychiatry. 2008 Jul; 41(4):163-5. 